\title{
Physical modeling of turbulent multiphase flow in a continuous casting steel mold
}

\author{
L. D. O. Campos ${ }^{1,2}$, P. Gardin ${ }^{1}$, S. Vincent ${ }^{2} \&$ J. P. Caltagirone ${ }^{3}$ \\ ${ }^{1}$ ArcelorMittal Maizières Global R\&D, France \\ ${ }^{2}$ Paris-Est Marne-la-Vallée University, France \\ ${ }^{3}$ TREFLE Laboratory, Bordeaux I University, France
}

\begin{abstract}
To understand the turbulent flow and mass transfer occurring inside a continuous casting (CC) mold machine, a multiphase CFD model is currently under development. This model will be used in subsequent studies to calculate the mass transfer coefficients of different species across the interface between liquid steel and slag. Those coefficients are necessary to predict slag composition and viscosity during the casting of steels. To validate the CFD model, some investigations to measure the velocity field in the region close to the interface, where the mass transfer between liquid steel and slag takes place, were carried out. Three fluids were considered, respecting main similarity criteria but not all: water to simulate the liquid steel, silicon oil to simulate the melted flux powder and air in the atmosphere domain. The velocity field was obtained by means of a Laser Doppler Anemometry (LDA) technique in the CC water model. Some parameters were evaluated regarding their effects on the interface behavior. Two different kinematic viscosities of oil were tested: 20 and $350 \mathrm{cSt}$. To evaluate the effect of water flow rate, it was set two flow rates: 1.6 and $2.0 \mathrm{~m}^{3} / \mathrm{h}$. Oil layer thickness was either $4 \mathrm{~mm}$ or $15 \mathrm{~mm}$. It was observed that slag viscosity has a strong effect on the flow near the interface: with the most viscous oil, the interface tangential velocity gradient in water was increased. The increase of shear stresses with the water flow rate was also quantified, which is important to better assess the possibility of slag entrainment during the casting.

Keywords: mass transfer, continuous casting, interface velocity.
\end{abstract}




\section{Introduction}

The continuous casting (CC) machine is responsible for more than $80 \%$ of the casted steel in the world. It allows the steel to be continued casted and its good performance is crucial to the productivity and to ensure the quality of the final steel product.

Liquid steel is injected by a submerged entry nozzle (SEN) in the mold with typical section of $1800 \times 220 \mathrm{~mm}^{2}$ during the casting. At the outlet of the nozzle the Reynolds number ranges 70000 , which produce high turbulent flows into the mold and close to liquid slag-steel interface.

Figure 1 gives a schematic view of the mold of a $\mathrm{CC}$ machine with the various phases present in the process.

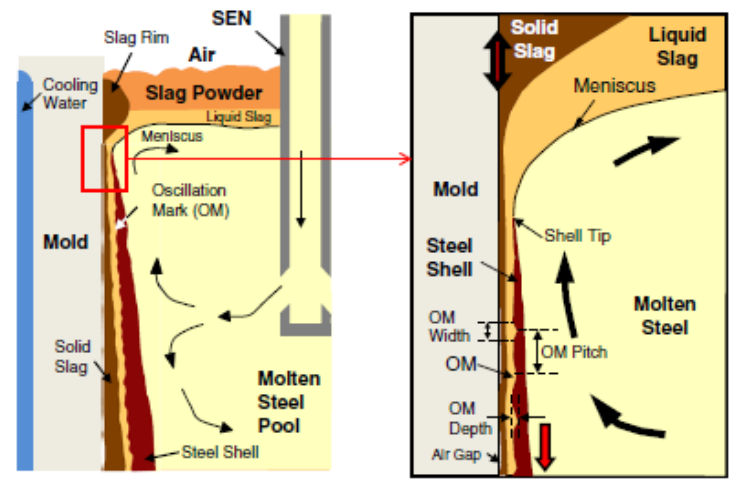

Figure 1: Different phases in a CC mold (Jonayat and Thomas [1]).

During the casting, the flux powder is continuously poured on the steel surface. At about $1000^{\circ} \mathrm{C}$ the flux is melted, forming a thin slag layer of a few millimeters which aims not only to protect the steel from oxidation and provide thermal insulation, but also to lubricate the mold. The liquid slag is dragged by the vertical oscillatory movement of the mold, forming a thin slag layer around the solidified steel shell, protecting the mold from contact and allowing the steel to flow down the mold.

When the molten slag layer is too viscous, the lubrication is deteriorated and the shear from the contact of the solidified skin of steel with the mold leads to a rupture of the shell, pinning the solid steel. If a sticking takes place, it causes a critical and dangerous problem also called as "break up", and the process has to be stopped. Then, it is of major importance to control the viscosity of the slag layer in order to ensure a good lubrication during the casting.

To guarantee a good lubrication the slag viscosity must be monitored. This can be achieved by controlling the slag composition, which changes during the casting. The new generation of advanced high-strength steels, for example, contains alloy elements that can be transferred to the slag, affecting its viscosity. Depending on the $\mathrm{Al}$ content, one of various alloy elements present in the steel, 
the $\mathrm{Al}_{2} \mathrm{O}_{3}$ on the slag can vary from about 3 to $35 \%$. Shahbazian et al. [2] showed that this increase in the alumina content of the liquid slag layer can increase up to five times the viscosity of the layer.

Since industrial trials are costly prohibitive, physical and mathematical simulations are used to predict the behavior of the fluids during the casting. Mishra et al. [3] provided a review of different physical and mathematical simulations of the CC process. Although many studies have been performed on this subject, most of them concern to the SEN design for optimum fluid flow into the mold for specific industrial cases [4]. A few works concerning the heat transfer have been investigated [5] and none for mass transfer in the CC machine until the present moment.

The accurate prediction of the interface is the first step to advance in the prediction of mass transfer in CC. Studies of the interface between the two immiscible fluids present during the casting started to come up recently. Kasai and Iguchi [6] evaluated, by water-model experiments, the behavior of the molten flux trapped by the vortex breakage using liquid paraffin and silicon oil to simulate different slag viscosities. They also studied the influence of different flow rates on the oil/water interface behavior. Pirker et al. [7] proposed a numerical approach to focus on local slag entrainment events within a global flow simulation in a tundish, which was later applied on $\mathrm{CC}$, originating the promising method called Lagrangian droplet model (LDM). The method is supposed to reproduce the vortex formation by local refinement of the mesh in the region with potential to vortex formation.

Mass transfer across the interface between two fluids is extensively studied in different fields of research. However, the studies usually consider a high Sc number with the order of a few hundreds. In steelmaking, the Sc numbers have the order of about 1000. Calmet and Magnaudet [8] showed that the most promising method to obtain the mass transfer coefficient at interfaces with high Sc numbers is the Large Eddy Simulation (LES) coupled with the Surface Divergence Method (SDM), where the mass transfer coefficient is given by

$$
k / u \propto\left(\gamma^{2}\right)^{1 / 4} S c^{-1 / 2}
$$

where $\gamma_{\text {is }}$ the surface divergence strength, and $u$ is the interfacial friction velocity, defined as $u=\left(\tau_{w} / \rho\right)^{\mathbf{1} / \mathbf{2}}$ where $\rho$ the fluid density and $\tau_{w}$ is the shear stress, usually given by the Newton's law of viscosity,

$$
\tau_{w}=\mu \frac{\partial U}{\partial y}
$$

Here, $\mu$ is the dynamic viscosity of the fluid, $\partial U / \partial y$ is the interface tangential velocity gradient and $y$ is the perpendicular distance from interface. Hence, it is of major importance to well measure the velocities in the vicinity of the interface between the fluids to be sure the model accurately calculates the shear stress.

Gardin et al. [9] coupled fluid dynamics and thermodynamics, evaluating the mass transfer across the liquid steel and slag interface in a CC. They also found 
the correlation for $k / u \propto S c^{-1 / 2}$ but scaling law was changed after a Sc number higher than 350. They proposed a new expression based on the surface divergence model, where $k / u \propto S c^{-3 / 2}$ for higher Sc numbers and emphasised the need of more tests to confirm the correlations.

To provide sufficient data to validate a mathematical model, a set of experiments were performed in water model, where the effect of different parameters on the interface behavior were analyzed. Only the experimental results will be presented and discussed in this paper.

In the next section, the physical apparatus will be described, as well as the materials used and parameters evaluated. After, some results will be presented and discussed for which a summary will be provided at the end of this paper.

\section{Experimental apparatus and evaluated parameters}

Figure 2 shows a water model used in the study. It is a CC mold built in acryl in full scale. It has a movable wall at the right side, which allows the study of the flow in the mold during the casting of steels with different widths. For the presented study, the width used was $720 \mathrm{~mm}$.

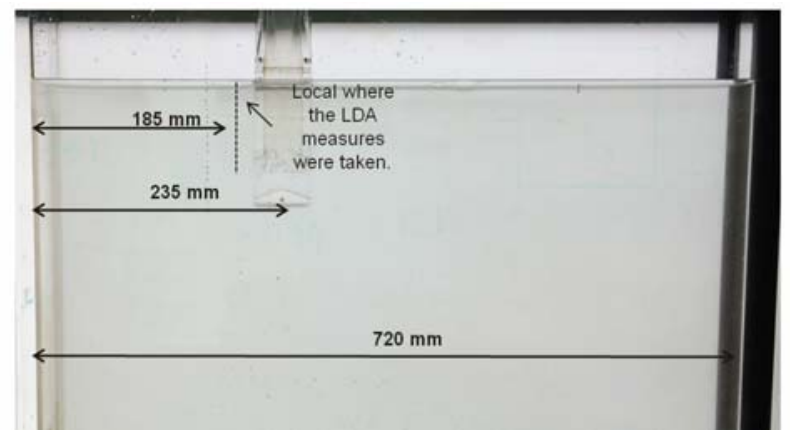

Figure 2: Physical apparatus and detailed measures of the studied configuration.

Water was used to simulate liquid steel. The flows of water and liquid steel are quite similar, as they have very similar kinematic viscosities $\left(v_{\text {water }}=1.0 \mathrm{cSt}\right.$ and $v_{\text {steel }}=0.8 \mathrm{cSt}$ ) [10]. Two silicon oils were used to simulate melted flux powder with two different kinematic viscosities.

The measures of the velocities were taken as close of the water/oil interface as possible. As the interest is to study the mass transfer, the velocity field must be measured in each fluid separately. To achieve this goal, the SEN was replaced to the left until a very stable interface was obtained.

The water flow rate, $Q$, through the SEN was controlled by an upper rod and the water level was kept constant during experiments with SEN depth $h_{w}=$ $68 \mathrm{~mm}$. 
The velocity measures were taken with two techniques. The Laser Doppler Anemometry (LDA) was used, at first, to take very precise measures at the water field. In a second step, the Particle Image Velocimetry (PIV) was used to have a more descriptive view of the flow in the region showed in Figure 2.

Measurement positions in the water model are shown in Figure 2 and the experimental conditions are detailed in Table 1 as well as the oil viscosities, $v$, and the oil layer thicknesses, $h_{o}$, used.

Table 1: Experimental conditions and physical properties.

\begin{tabular}{|c|c|c|}
\hline $\begin{array}{l}\text { Mold Size } \\
\text { Water Flow Rate, } Q \\
\text { Nozzle Depth, } \mathbf{h}_{\mathbf{w}} \\
\text { Oil layer thickness, } \mathbf{h}_{\mathbf{o}}\end{array}$ & & $\begin{array}{l}\mathrm{l}_{\mathrm{t}} \mathrm{mm} \\
00 \mathrm{~m}^{3} / \mathrm{h} \\
\mathrm{m} \\
5 \mathrm{~mm}\end{array}$ \\
\hline \multirow{2}{*}{$\begin{array}{c}\text { Physical Properties of the } \\
\text { Oil Layer }\end{array}$} & Silicon Oil A & $\begin{array}{l}\rho=950 \mathrm{~kg} \cdot \mathrm{m}^{-3} \\
v=20 \mathrm{cSt}\end{array}$ \\
\hline & Silicon Oil B & $\begin{array}{l}\rho=970 \mathrm{~kg} \cdot \mathrm{m}^{-3} \\
\nu=350 \mathrm{cSt}\end{array}$ \\
\hline
\end{tabular}

The velocities were measured during 10 minutes for each point in the LDA measures, and the mean velocities and random mean square (rms) were calculated. PIV measures were taken with 1000 pairs of frames every 0.2 seconds during 200 seconds.

\section{Experimental results and discussions}

\subsection{Water flow rate effect}

At the interface between oil and water, the effect of oil layer on interface behavior will increase with the increasing of the mass (evaluated as the thickness of the oil layer) and viscosity of the oil layer. This means that, the thicker and the more viscous the oil layer, the stronger is its effect on interface behavior, acting as a stagnant phase at the top of the water field. To impose its velocity as a result of the nonslip condition at the interface, the quasi-static behavior of the oil imposes an abrupt change in the water flow velocity, generating a pseudoboundary layer as schematically shown in Figure 3.

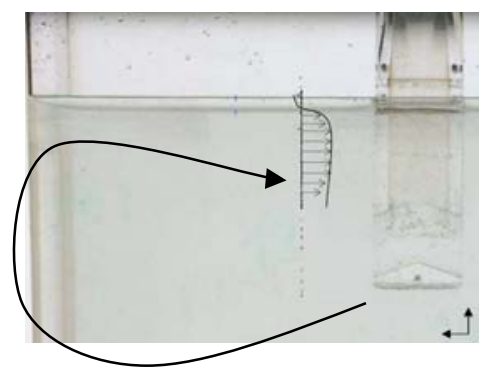

Figure 3: Schematic view of the horizontal velocity profile with the boundary layer close to the interface. 
For a $15 \mathrm{~mm}$ thick oil layer, the water flow at the interface is deeply affected by the oil motion, which has only cyclic horizontal movements with very low mean velocity and relatively high fluctuations.

Measurements have shown that, keeping the viscosity of the oil layer, an increase in the water flow will increase the horizontal velocities in the bulk flow, but will have no effect on the vertical component, as shown in Figures 4 and 5.

Figure 4 also shows that, keeping the oil layer configuration, an increase in the water flow rate will increase the parallel velocity gradient near the interface. So, the higher the velocity field, the higher will be the velocity gradient at the water field.
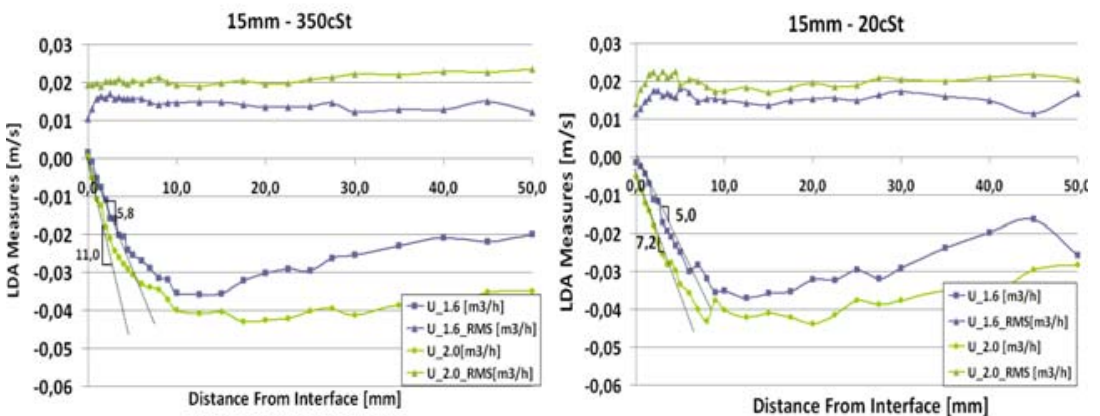

Figure 4: LDA measures of the interface tangential velocity components, $U$, from 4 different configurations.
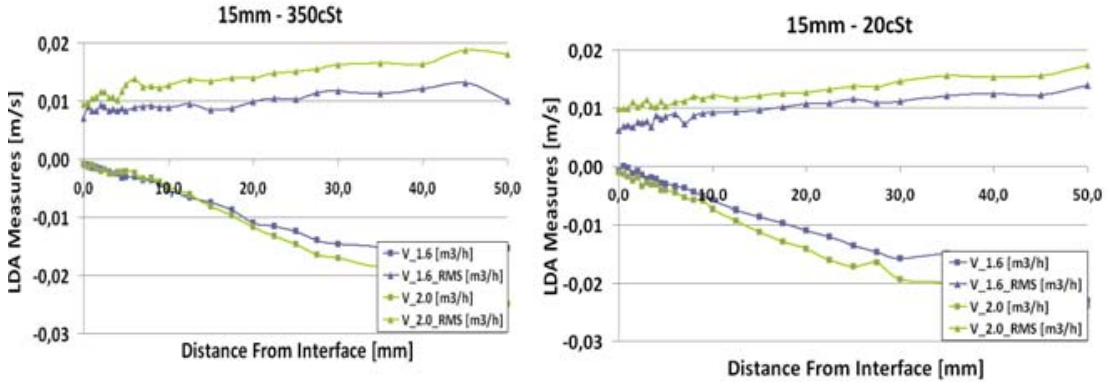

Figure 5: LDA measures of the interface perpendicular velocity components, $V$, from 4 different configurations.

Here, the main difference of the interface boundary layer from a wall boundary layer is the rms value which is null in the second case but keeps an almost stable value in the interface between two liquids. Even though the mean tangential velocities at the liquid/liquid interface are slim to none, there is, indeed, some oscillatory movement, which explains the rms values higher than the mean velocities. 
In both, interface tangential and perpendicular velocity components, the increase of the water flow increased the fluctuations in the velocity values (rms), which suggests that the turbulence intensity should be proportional to the flow rate into the mold.

\subsection{Oil layer viscosity effect}

The viscosity plays an important role for the interface behavior. Figure 6 shows that, if the velocities measured with the same water flow rate are compared, the interface tangential velocity rates are higher in the cases with higher viscosities. This tendency is reinforced when the water flow increases.
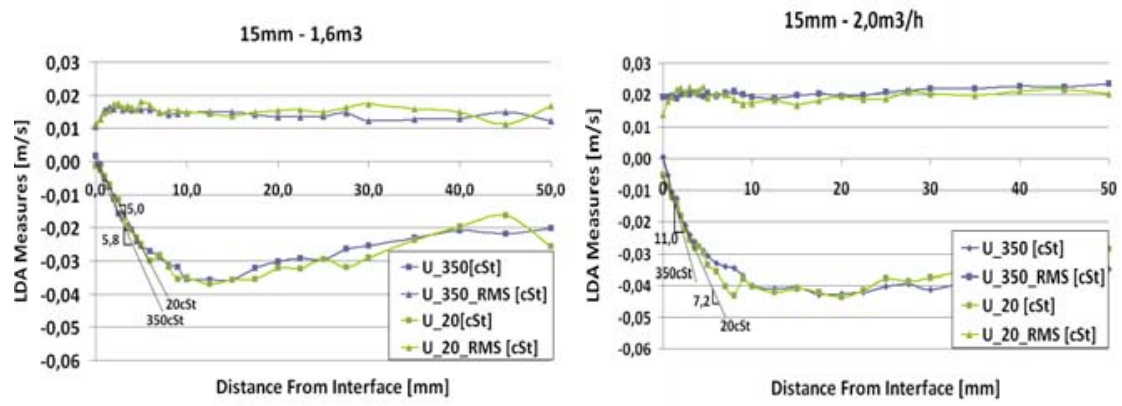

Figure 6: LDA measures of the interface tangential velocity components, $U$, with different oil viscosities.

The results show that an oil layer more viscous will generate higher shear stresses. When the shear stresses are higher than the surface tension between those fluids, oil entrapment can occur. The same occurs in steelmaking processes, where slag entrainment can cause loss of quality by generating slag inclusions, which are detrimental to the steel performance in its final application. Any influence in the flow far from the interface was verified by changing the oil viscosity.

A negligible effect of the oil viscosity was observed in the interface perpendicular velocity component, as shown in the Figure 7 below:
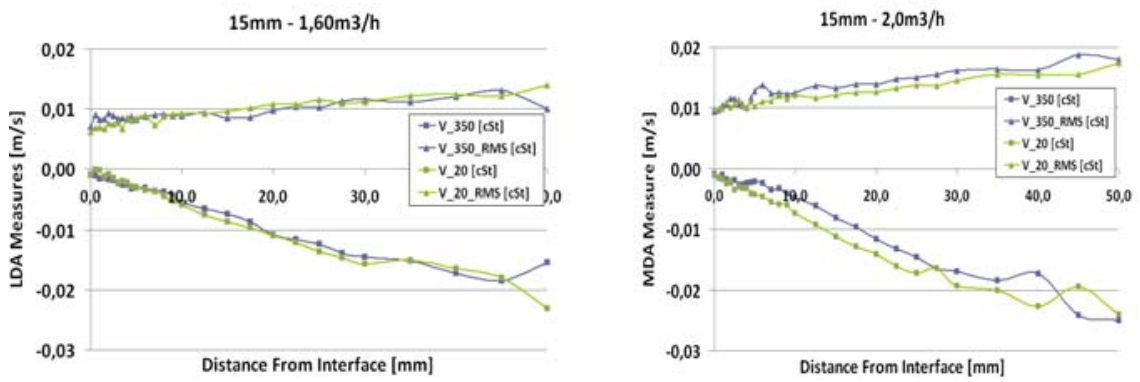

Figure 7: LDA measures of the interface perpendicular velocity components, $V$, with different oil viscosities. 


\subsection{Oil layer thickness effect}

The velocity fields measured with $4.0 \mathrm{~mm}$ and $15 \mathrm{~mm}$ oil layer thickness were compared. The interface tangential velocity gradient is higher in the region near to the interface of the thinner layer for the lowest water flow rate, as shown in Figure 8:

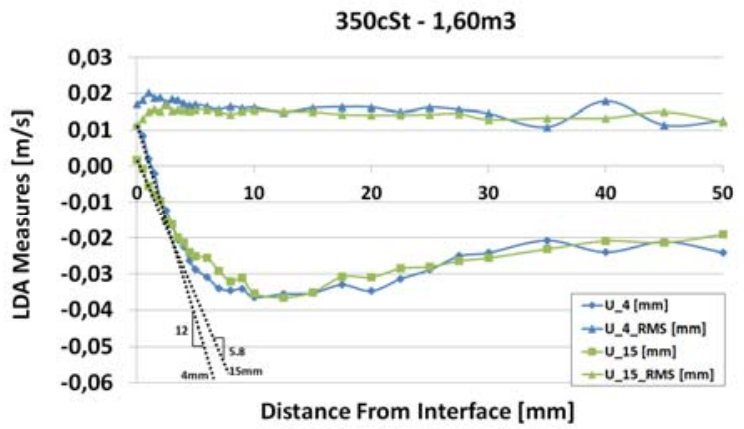

Figure 8: LDA measures of the interface tangential velocity components, $U$, with different oil layer thickness and $1.6 \mathrm{~m}^{3} / \mathrm{h}$ water flow rate.

This happens because at the positions where the measures were taken, for the $4.0 \mathrm{~mm}$ oil layer thickness, there is a recirculation zone at the left side of the SEN, which is schematically displayed in Figure 9 (left).

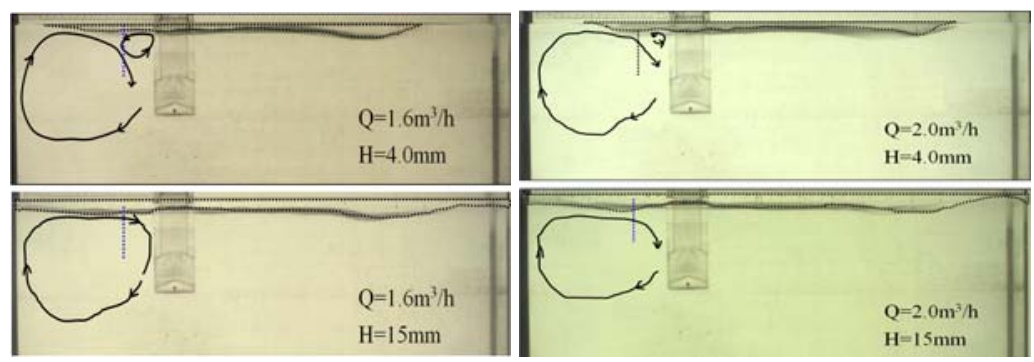

Figure 9: Schematic view of the flow at the regions where the velocity measures were taken.

The reason why there is a recirculation zone with the thinner oil layer is not yet completely clear, but it was observed that, when the oil layer is not sufficiently thick, the water flow pushes the oil layer away from the lateral walls, even with weak water flow rates, creating a region of air/water contact, which may influence the flow, anticipating the recirculation of the flow for a region closer to the walls. If the flow is higher, e.g. $2.0 \mathrm{~m}^{3} / \mathrm{h}$, the water free surface may not affect the flow, and no recirculation zone was observed, as shown by the LDA measures in Figure 10. 


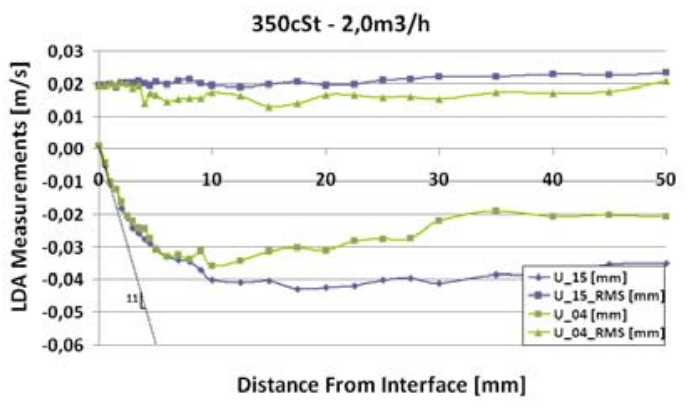

Figure 10: LDA measures of the interface tangential velocity components, $U$, with different oil layer thickness and $2.0 \mathrm{~m}^{3} / \mathrm{h}$ water flow rate.

Figure 10 shows that, for the highest water flow rate tested, the horizontal velocity gradients close to the interfaces are the same, no matter the thickness of the oil layers. However, the water free surface appearing in an insufficiently thick layer seems to attenuate the horizontal velocities in the bulk flow. That can be explained by the flow behavior observed, which was schematically illustrated above, in Figure 9 (right).

In Figure 9, one can note that with high water flow rates the water free surface is increased and the recirculation zone is pushed towards the SEN to a region where the LDA measures could not detect. With that configuration, the interface tangential velocity components of the fluid are lower than the same velocity field measured with the thicker oil layer as well as its rms values.

The difference in the measures taken with the recirculation zone (low water flow rate and thinner oil layer) and without it (high water flow rate, i.e. $2.0 \mathrm{~m}^{3} / \mathrm{h}$ ) can be observed in the interface perpendicular velocity component:

There are more perturbations in the recirculation zone and the mean vertical velocities measures vary in the interface vicinity as well as the rms, as shown in Figure 11(a).

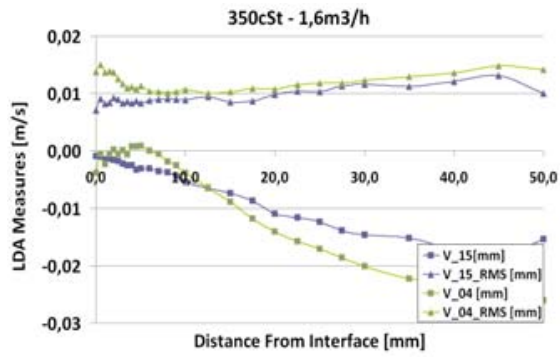

(a)

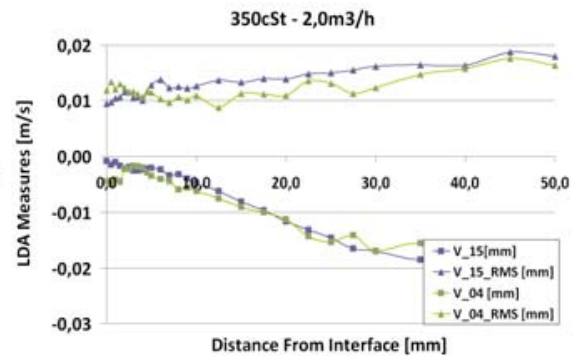

(b)

Figure 11: LDA measures of the interface perpendicular velocity components, $V$, with different oil layer thickness and $1.6 \mathrm{~m}^{3} / \mathrm{h}(\mathrm{a})$ and $2.0 \mathrm{~m}^{3} / \mathrm{h}(\mathrm{b})$ water flow rate. 
The PIV confirmed the existence of the recirculation zones, as shown in Figure 12.

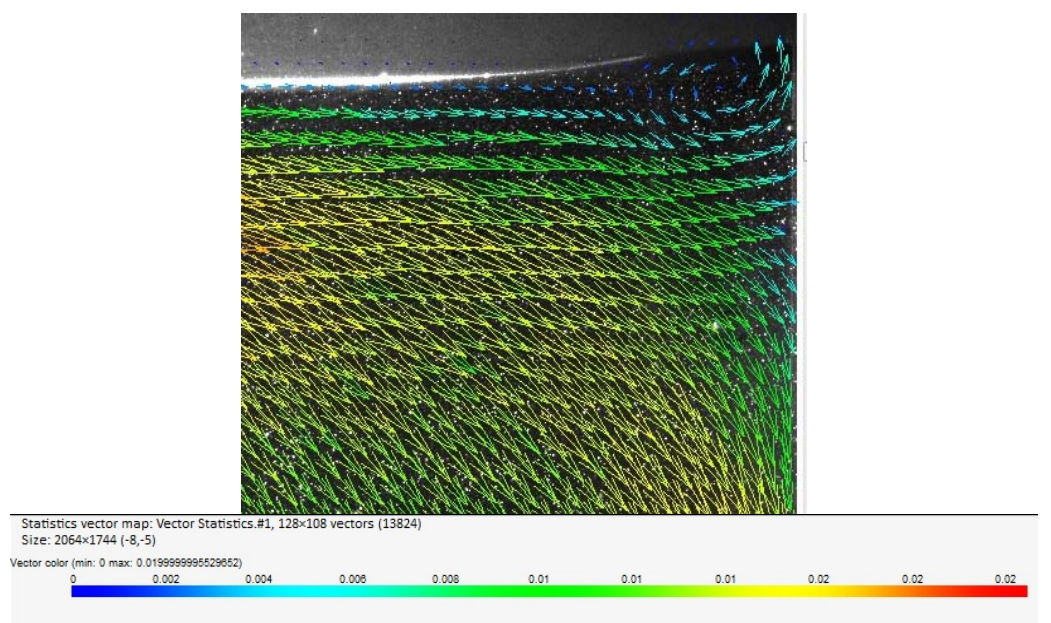

Figure 12: Velocity field obtained by PIV, showing the recirculation zone close to the SEN.

\subsection{Conclusions}

During the physical experiments, it was analyzed the effect of the water flow rate, oil layer viscosity and oil layer thickness on the interface behavior and shear stresses were analyzed and evaluated by Eq. (2).

The values of the measured shear stresses are summarized in Table 2:

Table 2: Shear stresses for each configuration.

\begin{tabular}{cccc}
\hline $\begin{array}{c}\text { Water } \\
\text { Flow Rate } \\
\left(\mathrm{m}^{3} / \mathrm{h}\right)\end{array}$ & $\begin{array}{c}\text { Oil Layer } \\
\text { Viscosity } \\
(\mathrm{cSt})\end{array}$ & $\begin{array}{c}\text { Oil Layer } \\
\text { Thickness } \\
(\mathrm{mm})\end{array}$ & $\begin{array}{c}\text { Shear Stress } \\
\left(10^{-3} \mathrm{~kg} / \mathrm{m} . \mathrm{s}^{2}\right)\end{array}$ \\
\cline { 2 - 4 } 1.6 & 20 & 15 & 0.095 \\
\hline & 350 & 4 & 4.080 \\
& 20 & 15 & 1.972 \\
\hline \multirow{2}{*}{2.0} & 350 & 15 & 0.137 \\
& & 4 & 3.740 \\
& & 15 & 3.740 \\
\hline
\end{tabular}

In summary, the influences observed were:

\section{Water flow rate}

- When there is no water free surface $(15 \mathrm{~mm})$, an increase in the water flow rate will increase the shear stress at the interface. 
- Increasing the water flow rate will increase the fluctuation of the velocity field close to the interface and in the bulk flow, no matter the oil layer thickness.

\section{Oil layer viscosity}

- Increasing the oil layer viscosity will increase the shear stresses at the interface.

- The oil layer viscosity does not have any influence on the bulk flow neither in the velocity field fluctuations.

\section{Oil layer thickness}

The oil layer thickness has a huge effect on the flow at the upper part of the mold and this effect must be analyzed according to the water flow rate:

- Low water flow rates:

When there is a low flow rate, the oil layer thickness has no effect on the mean velocities in the bulk flow.

However, for a thin layer, a recirculation zone is created and the tangential interface velocity gradient is higher than for a thick layer (Figure 9). This can be explained by the fact that in the regions of the measures, there is a change in the horizontal velocity sense.

- High water flow rates:

When the flow is sufficiently high, the recirculation zone is compressed close to the SEN or even suppressed. Without the presence of that phenomenon, the horizontal velocity rate at the interface is exactly the same (Figure 10), and the oil layer thickness has no effect on it. However, with a thin layer, the free surface changes the shape of the flow and the horizontal velocity component is weaker far from the interface.

The experimental part of the study was successfully finished and the data needed to validate a future CFD model is provided.

\section{References}

[1] Jonayat, A. \& Thomas, B.G. Transient Thermo-fluid Model of Meniscus Behavior and Slag Consumption in Steel Continuous Casting. The Minerals, Metals \& Materials Society and ASM International 2014.

[2] Shahbazian, F., Sichen, D. \& Seetharaman, S. The Effect of Addition of $\mathrm{A} 12 \mathrm{O} 3$ on the Viscosity of $\mathrm{CaO}-$ "FeO"-SiO2-CaF2 Slags. ISIJ International, Vol. 42 (2002), No. 2, pp. 155-162.

[3] Mishra, P., Ajmani, S.K., Kumar, A. \& Shrivastava, K.K. Review article on physical and numerical Modeling of SEN and mold for continuous slab casting. International Journal of Engineering Science and Technology (IJEST). Vol. 4, No. 5, May 2012.

[4] Brummayer, M., Gittler, P. \& Watzinger, J. Stabilization of unsteady turbulent flow in the mold region of a wild slab caster by submerged entry nozzle design optimization with CFD. Second International Conference on 
CFD in the Minerals and Process Industries. CSIRO, Melbourne, Australia. 6-8 December 1999.

[5] Yuan, Q., Zhao, B., Vanka, P. \& Thomas, B.G. Study of Computational Issues in Simulation of Transient Flow in Continuous Castin. Steel research int. 76 (2005) No. 1.

[6] Kasai, Norifumi and Iguchi, Manabu. Water-model Experiment on Melting Powder Trapping by Vortex in the Continuous Castin Mold. ISIJ International. $\mathrm{N}^{\circ}$ 7, 2007, Vol. 47.

[7] Pirker, S., Reiter, J. \& Ernst. Sharpening the modelling of slag entrainment during tundish pouring, G. N8, s.1. : Steel Research Journal, 2010, Vol. 81.

[8] Calmet, M., \& Magnaudet, J. High-Schmidt number mass transfer through turbulent gas-liquid interfaces. International Journal of Heat and Fluid Flow 19, pp. 522-532. 1998.

[9] Gardin, P., Gauthier, S. \& Vincent, S. Prediction of Mass Transfer between liquid steel and slag at continuous casting slag mold. 10th International Conference on CFD in Oil \& Gas, Metallurgical and Process Industries. 2014.

[10] Yuan, Q., Thomas, B.G. \& Vanka, S.P. Study of Transient Flow and Particle Transport in Continuous Steel Caster Molds: Part I. Fluid Flow. Metallurgical and Materials Transactions B. Volume 35B, August 2004. 